\title{
GAZELA: ASESOR DIGITAL DE REDES SOCIALES PARA ADOLESCENTES
}

\author{
Alberto Isasi-Andrieu, Ángel López-Carrera, Pilar Ruiz-Ibáñez e Iñaki Angulo- \\ Redondo
}

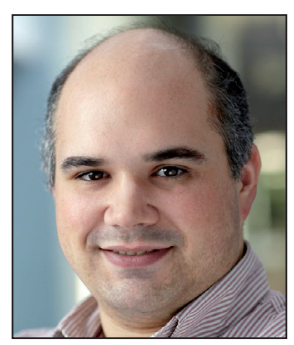

Alberto Isasi-Andrieu, ingeniero industrial e ingeniero en automática y electrónica industrial por la Universidad del País Vasco y diploma de estudios avanzados en ingeniería de electrónica y control, es doctorando, investigando modelos de color bioinspirados para la segmentación automática de imágenes digitales. Es investigador en el centro tecnológico Tecnalia, trabajando en aplicaciones de visión artificial para las TICs y el sector industrial.

Área de Computer Vision División ICT-European Software Institute, Tecnalia Parque Tecnológico de Bizkaia Ibaizabal Bidea, Edif. 202. 48170 Zamudio, España alberto.isasi@tecnalia.com

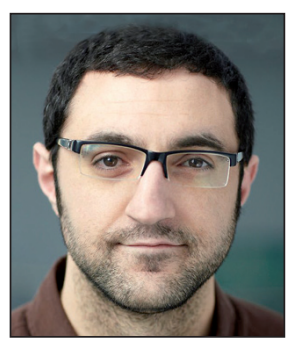

Ángel López-Carrera es técnico superior en administración de sistemas informáticos. Es técnico programador de Tecnalia desde hace 11 años y en la actualidad trabaja en proyectos de investigación en el desarrollo de aplicaciones de ayuda a la toma de decisiones aplicando Business Intelligence, aplicaciones relacionadas con las redes sociales de internet y algoritmos ágiles para la indexación y recuperación de imágenes médicas por similitud visual.

Tecnalia, Área de Computer Vision angel.lopez@tecnalia.com

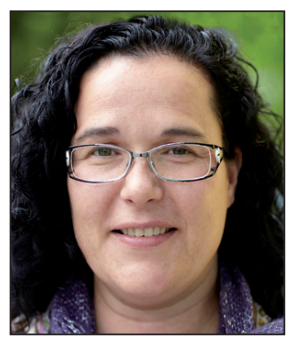

Pilar Ruiz-Ibáñez, licenciada en geografía e historia por la Universidad de Granada, es investigadora en el Área de Service Industry de Tecnalia R\&I. Antigua responsable del Centro de Documentación de Robotiker. Es directora del proyecto de fusión de Gestión del Conocimiento de Tecnalia R\&I.

Tecnalia, Área de Service Industry pilar.ruiz@tecnalia.com

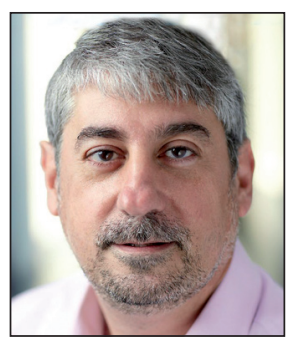

Iñaki Angulo-Redondo es licenciado en informática por la Univ. de Deusto. Tiene más de 20 años de experiencia en centros tecnológicos habiendo participado en proyectos de I+D sobre aplicaciones empresariales de tecnologías avanzadas de la información y comunicación, tanto nacionales como europeos. Es gerente de mercado en el Área de Optimización, Modelización y Analítica (Óptima) de Tecnalia.

Tecnalia, Área Óptima inaki.angulo@tecnalia.com

\section{Resumen}

El uso de las redes sociales se ha masificado compartiéndose a través de ellas millones de archivos, vídeos y fotografías al día y sustituyendo a otros sistemas de comunicación más tradicionales como el teléfono, e-mail y sms. Sin embargo, muchas veces se utilizan estos medios arbitrariamente desconociendo sus repercusiones posteriores. Por ello, nace Gazela, una plataforma que trata de proporcionar a la sociedad en general y a los adolescentes en particular, las herramientas necesarias que les permitan garantizar su privacidad a la hora de participar en las redes sociales. Su actividad se centra en tres elementos principales: servicios de vigilancia, servicios de protección, y servicios de asesoramiento jurídico.

\section{Palabras clave}

Redes sociales, Bullying digital, Cyberbullying, Privacidad, Semántica, Biometría, Reconocimiento facial, Asesoramiento legal, Suplantación de identidad, Pederastia, Vigilancia, Protección, Proyecto, Procesamiento de imagen. 
Title: Gazela: social networks' digital advisor for teenagers

\begin{abstract}
Social networks are becoming overcrowded. Every day people share millions of files, videos and pictures, and the massive use of social networks is replacing other communications channels such as telephone, e-mail or sms. However, these networks are often used without knowing the possible repercussions. Gazela is a platform with the aim to provide the general society and especially teenagers with the necessary tools to allow them to guarantee their privacy while using social networks. Gazela's activity is focused on three main elements: surveillance services, protection services and legal advising services.
\end{abstract}

\title{
Keywords
}

Social networks, Digital bullying, Cyberbullying, Privacy, Semantics, Facial recognition, Biometrics, Legal advising, Identity theft, Child abuse, Surveillance, Protection, Project, Image processing.

Isasi-Andrieu, Alberto; López-Carrera, Ángel; Ruiz-Ibáñez, Pilar; Angulo-Redondo, Iñaki. "Gazela: asesor digital de redes sociales para adolescentes". El profesional de la información, 2012, septiembre-octubre, v. 21, n. 5, pp. 514-519. http://dx.doi.org/10.3145/epi.2012.sep.11

\section{Introducción}

Es jueves y los alumnos de 10 de ESO de un colegio van de excursión al Museo Chillida Leku para contemplar la obra de este artista vasco. Después del paseo por las distintas salas y de atender a las explicaciones de los guías, los chavales disponen de tiempo para comer, tomar fotografías y hacer bromas con sus compañeros. Todo transcurre en un ambiente de cordialidad, hasta que un grupo decide gastarle una broma a Susana. Ya por la noche, Mikel, que ha grabado la broma en su móvil, la sube a Tuenti para que pueda verla el resto de la clase. Al cabo de unos días la policía se persona en el colegio para recabar información en relación a una denuncia realizada por los padres de Susana como consecuencia de la publicación del vídeo grabado por Mikel en YouTube.

Esta situación es ficticia pero bien podría darse en cualquier colegio, biblioteca o centro formativo, y muestra dos aspectos de las consecuencias que tiene la participación de los adolescentes en las redes sociales. Por una parte Susana ve amenazada su privacidad en el vídeo colgado por Mikel, y por otra Mikel no ha sido consciente de que, lo que para ellos era una broma, para Susana supone una situación muy desagradable. A esto hay que añadir que situaciones como el acoso a través de las redes sociales, o cyberbullying, la suplantación de identidad por pederastas, o la recepción de mensajes ofensivos o pornográficos, están siendo cada vez más habituales. Y los niños y jóvenes son los más vulnerables a este tipo de amenazas.

Con la introducción de las nuevas tecnologías en nuestro día a día, cada vez es más fácil acceder a los servicios online. Redes sociales como Facebook, Tuenti o Twitter, o las profesionales como LinkedIn o Yammer, son cada vez más usadas por miles de personas para comunicarse y publicar información que puede llegar a ser accesible por cientos o incluso miles de personas.

Sin embargo, muchas veces se infringe una serie de derechos y leyes asociados a los contenidos que se comparten, como las fotografías de amigos que se suben a la Red sin los permisos de los que en ellas aparecen.
Además, los menores usan cada vez más internet para compartir información y establecer relaciones entre ellos, sin ser conscientes de que aspectos de su intimidad y privacidad se puedan ver vulnerados por navegar en estas webs sociales. Esta información puede ser utilizada por terceros sin consentimiento $y$, en ocasiones, como medio de chantaje, coacción y agresión física o moral. Es por ello que las redes sociales se han convertido en un nuevo espacio para el bullying escolar.

\section{Las redes sociales se han convertido en un nuevo espacio para el bullying escolar}

Para abordar este problema nace Gazela, un proyecto de investigación en curso en el área de las tecnologías de la información, cuyo objetivo es la elaboración de una plataforma que proporcione, a la sociedad en general y a los adolescentes en particular, las herramientas necesarias para garantizar su privacidad a la hora de participar en las redes sociales. En él colaboran Entelgy Ibai, empresa de tecnologías de la información con experiencia en la búsqueda y localización de información masiva; Seinale, un despacho de abogados especializado en los aspectos legales relacionados con las tecnologías de la información; el centro de formación Irungo La Salle; y el centro tecnológico Tecnalia con experiencia en tecnologías de internet y en interpretación de información multimedia.

\section{Antecedentes}

En el Protocolo de actuación escolar ante el cyberbullying elaborado por Emici (Equipo Multidisciplinar de Investigación sobre Cyberbullying) para el Gobierno Vasco (Protocolo Emici, 2011) se describen las principales características del ciberacoso en el entorno escolar. Puede aparecer en tres formas:

- hostigamiento mediante fotos o vídeos;

- exclusión, cuando se usan entornos públicos para acosar 
o hacer comentarios que provocan una respuesta expansiva de rechazo de una persona;

- manipulación, cuando se utiliza la información encontrada para difundirla de modo no adecuado.

En el trabajo de Emici se incluyen 10 consejos (Decálogo, 2011) que se deberían tener en cuenta para una navegación "segura" en las redes sociales. Utilizando la herramienta Gazela se pueden seguir muchos de los consejos descritos.

\section{Decálogo para frenar el acoso}

1. Pide ayuda a tu padre o a tu madre

2. Nunca respondas a las provocaciones

3. No hagas presunciones

4. Evita aquellos lugares en los que sufres acoso

5. Protege tu privacidad:

a) Evita intrusos

b) Depura la lista de contactos

c) Reconfigura las opciones de privacidad

d) Comprueba qué cuentan de ti on-line

e) Repasa la información qué publicas y quién puede acceder

f) Comunica qué tipo de informaciones/imágenes no deseas

g) Ejerce tu derecho a la protección de datos personales

6. Guarda las pruebas del acoso

7. Trata de hacer saber a quienes te acosan que lo que están haciendo te molesta

8. Trata de hacerles saber que lo que están haciendo es perseguible por la ley

9. Deja constancia de que estás en disposición de presentar una denuncia

10. Toma medidas legales

Figura 1. Decálogo para frenar el acoso

Gazela proporciona herramientas que permitan garantizar la privacidad y seguridad para participar en las redes sociales

\section{Qué es Gazela}

Una herramienta de sensibilización, educación, protección y orientación de adolescentes de entre 12 y 18 años (ESObachillerato) que hacen uso de las redes sociales para compartir información.

Con sensibilizar se pretende conseguir una dinámica de buenas prácticas en el uso de las redes sociales, para que tengan presente cosas como pedir la autorización para publicar imágenes en la que aparecen otras personas, no invadir la privacidad de otros usuarios, evitar enviar información de carácter sensible, etc.

También es necesario educar a los usuarios en las implicaciones legales que tienen sus acciones en la Red y proporcionarles información de cómo protegerse ante posibles amenazas o acosos de sus contactos y orientarles en cómo proceder ante ellos.

Existen varias herramientas comerciales que tratan con algunas de estas problemáticas como NetNanny (NetNanny, 2012), los filtros de control parental (Control parental de Windows7, 2012; Control parental de Firefox, 2012; ConsumerSearch, 2012), o servicios como el ReputationDefender (Reputation manager) que rastrean la Red para encontrar información sobre una persona. Pero Gazela no trata de aplicar un control estricto de las acciones del usuario, sino aconsejar y establecer unas "buenas prácticas" que ayuden a educar en el uso de las redes sociales.

\section{Arquitectura}

Desde un punto de vista tecnológico, Gazela se basa en tres elementos principales:

- Servicios de vigilancia: orientados a recabar la información publicada por el usuario, comprobando que está dentro del marco legal en cuanto a contenidos, permisos de publicación, etc.

- Servicios de protección: para detectar y alertar si se ha publicado algo que pueda ser ofensivo para el usuario, notificándoselo al infractor.

- Servicios de asesoramiento jurídico: información básica sobre cómo actuar ante determinadas situaciones que puedan darse en las redes sociales, como acoso, chantaje o cyberbullying.

La plataforma se basa en tres elementos principales: servicios de vigilancia, de protección, y de asesoramiento jurídico

Existe una serie de servicios centralizados en un servidor, donde se realizan las labores más "pesadas" que no requieren de la intervención directa del usuario (figura 2).

- miAbogado: asesora sobre el procedimiento a seguir ante determinadas situaciones que pueden darse durante el

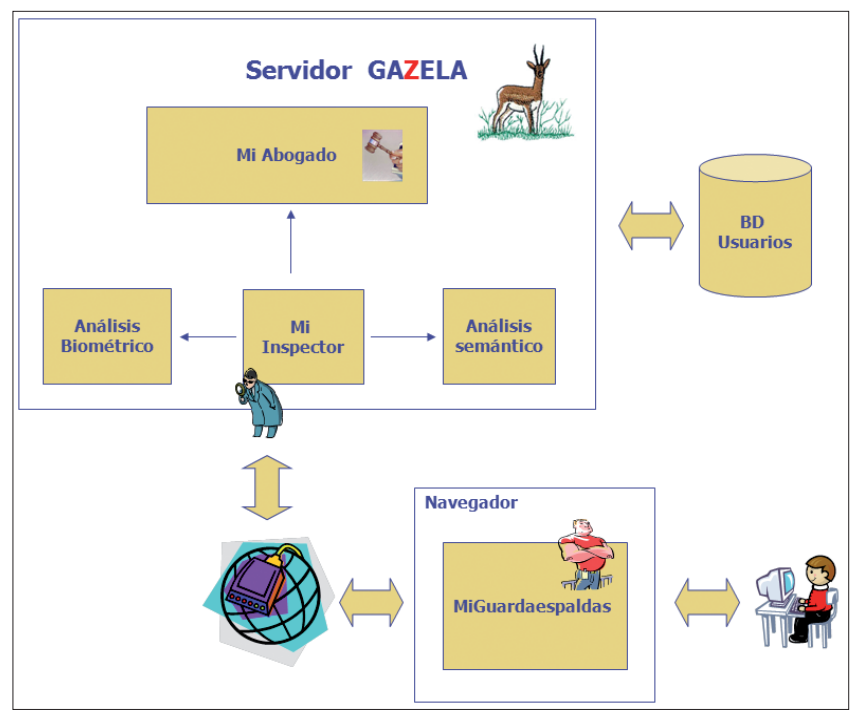

Figura 2: Servidor Gazela 
uso de las redes sociales. Ofrece información sobre las posibles consecuencias de no cumplir las normas a la hora de publicar una información determinada, o consejos sobre las acciones a tomar al recibir mensajes que puedan atentar contra la privacidad de una persona. No pretende sustituir a los bufetes de abogados, sino proporcionar una base inicial con la que acudir a éstos en caso de que fuera necesario.

- milnspector: módulo central de actuación de Gazela, encargado de coordinar el funcionamiento del resto de módulos. Analiza los contenidos publicados en la Red, examinando tanto la información escrita (mediante análisis semántico) como la contenida en imágenes y fotografías (mediante análisis biométrico). Para ello cuenta con la información almacenada en la base de datos (BD) de usuarios.

- Análisis biométrico: sistema experto de identificación de personas en imágenes, basado en el reconocimiento de patrones biométricos. Cuando se publican fotografías en las redes sociales, este módulo detecta a la persona o personas que aparecen en la imagen haciendo uso de la información biométrica recogida en la BD de usuarios.

- Análisis semántico: analiza lo que se publica en las redes sociales para detectar posibles contenidos ofensivos o protegidos que hayan sido escritos, ya sea de forma consciente o inconsciente. Este tipo de análisis busca y analiza los textos para encontrar una serie de estructuras gramaticales definidas a priori y, en caso de encontrarlas, avisa al usuario que las ha escrito recomendándole que corrija los contenidos.

- miGuardaespaldas: Establece la "relación" con el usuario, esto es, la interfaz mediante la cual se puede configurar Gazela para que supervise de forma adecuada la navegación. A través de este módulo se envían notificaciones y avisos al usuario, así como las recomendaciones del abogado. También se encarga del proceso de registro de nuevos usuarios, con su perfil sociológico, datos privados, información sensible, contacto, etc., así como de la generación del perfil biométrico, necesario para el reconocimiento facial.

- Base de datos de usuarios: almacena toda la información necesaria para el funcionamiento de los módulos que componen Gazela.

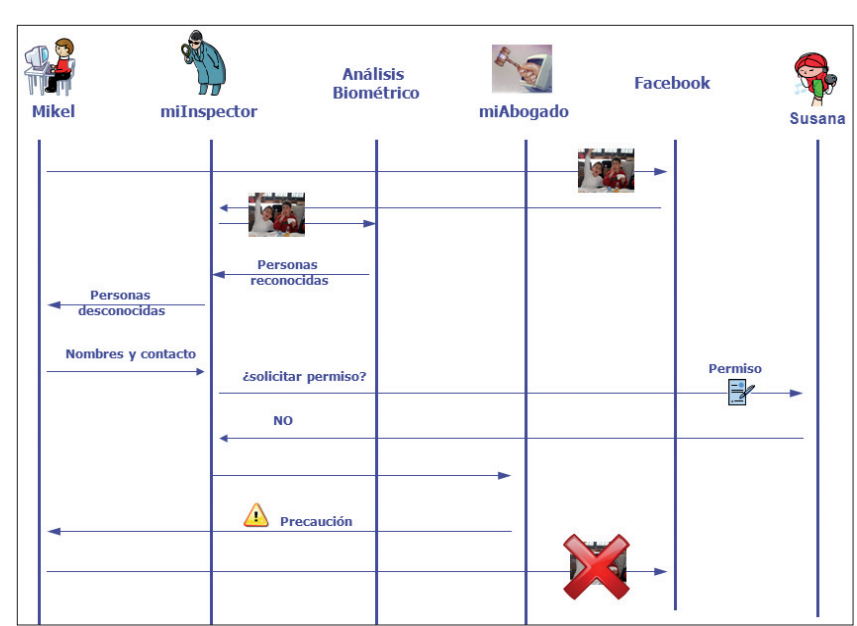

Figura 3. Secuencia de Gazela para identificar personas y pedir autorización

\section{Integración en redes sociales}

Inicialmente se ha centrado en Facebook, por ser la red social con mayor número de usuarios y donde mayor cantidad de información compartida existe actualmente (especialmente fotografías). Además, Facebook emplea Graph API (GraphAPI, 2012), un software muy maduro que hace posible el acceso a toda esa información por parte de aplicaciones desarrolladas por terceros, como es el caso de Gazela. Milnspector es el módulo encargado de conectarse a la red social para solicitarle toda la información que necesite.

Elementos de la plataforma Gazela: miAbogado, milnspector, análisis biométrico, análisis semántico, miGuardaespaldas y bdd de usuarios

A día de hoy, se han implementado con éxito las siguientes funciones:

- autenticación automática, con la que se logra el acceso a la información contenida en las cuentas de Facebook de los usuarios registrados en Gazela;

- acceso periódico a las nuevas publicaciones generadas por el usuario y sus amigos;

- análisis del contenido de dichas publicaciones en busca de infracciones, agresiones y datos protegidos haciendo uso del analizador semántico;

- acceso periódico a las imágenes nuevas publicadas;

- identificación de las personas que aparecen en las fotografías mediante el analizador biométrico;

- comunicación con miGuardaespaldas y miAbogado;

- registro en la base de datos de todas las infracciones, agresiones y datos protegidos encontrados durante los procesos de análisis.

En la siguiente fase de desarrollo, milnspector será capaz de revisar las publicaciones de los usuarios catalogadas por Gazela como "ilícitas" para comprobar que sus contenidos hayan sido corregidos o borrados y verificará que se hayan recibido las autorizaciones por parte de todas las personas que aparezcan en las fotografías subidas a Facebook.

\section{Biometría facial}

El reconocimiento facial es una de las modalidades de reconocimiento biométrico más difundido, al ser poco intrusiva y de alta aceptabilidad entre los usuarios (De-Luis-García; Alberola-López; Aghzout; Ruiz-Alzola, 2003). De hecho, en un estudio comparativo entre 6 modalidades biométricas, la basada en reconocimiento facial obtuvo la mejor puntuación (De-Luis-García; Alberola-López; Aghzout; Ruiz-Alzola, 2003).

A pesar de los continuos avances, los sistemas de reconocimiento facial aún no son completamente robustos a cambios de iluminación, pose, expresión u oclusiones parciales del rostro. Es en estos problemas donde se centran gran cantidad de investigaciones tanto a nivel universitario como comercial para conseguir sistemas biométricos más fiables (Face recognition homepage, 2012). En esta línea se han 
desarrollado numerosas técnicas basadas en máquinas de vectores soporte (SVM) (Guodong, 2000), boosting (Kong et al., 2005), modelos ocultos de Markov (HMM) (Nefian; Hayes, 1998) y redes neuronales (Latha; Ganesan; Annadurai, 2009) para mejorar las tasas de reconocimiento. Sin embargo, en el sistema Gazela se ha optado por enfocar el problema desde un nuevo punto de vista, mejorando la calidad de la imagen antes de la etapa de reconocimiento.

Se ha implementado un algoritmo utilizado habitualmente para la etapa de reconocimiento, y se han centrado los esfuerzos en robustecer y mejorar la fotografía a analizar mediante algoritmos de procesamiento de imagen. Para ello se están aplicando algoritmos de corrección de luminosidad, lo que permite aumentar la robustez frente a brillos o deslumbramientos (Chen et al., 2005). Para tratar el problema de la "pose", se está trabajando en enrolado múltiple, de modo que se tengan varios perfiles de un usuario que cubran perfil izquierdo, derecho e imagen frontal, para así obtener una tasa mayor de reconocimientos faciales correctos.

Otro aspecto a tener en cuenta es que el tipo de imágenes esperadas son grupos de personas en situaciones muy variadas y en localizaciones no controladas, y que se necesitará un entrenamiento continuo dado que el aspecto de las personas va evolucionando, lo que implica que el perfil biométrico de cada uno de los usuarios debe ir aprendiendo los nuevos rasgos de cada una de las personas enroladas en el sistema.

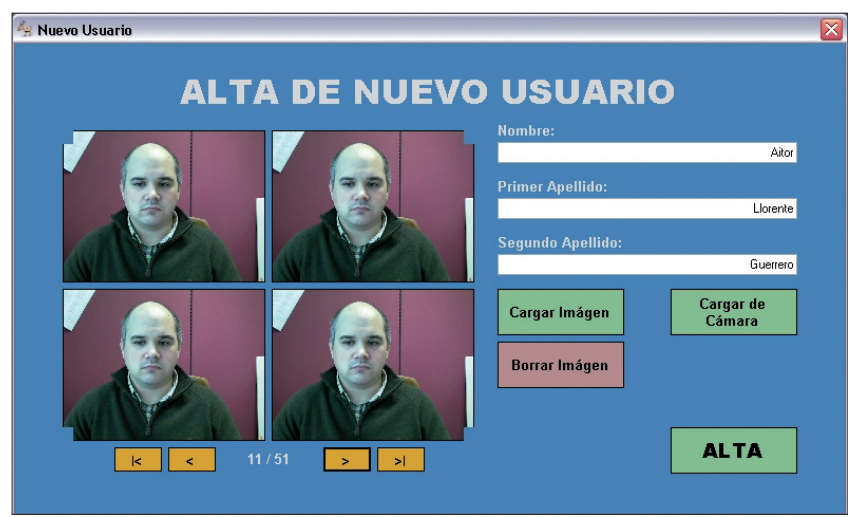

Imagen 1. Interfaz para realizar el alta biométrica de los usuarios mediante webcam

\section{Análisis semántico}

Las herramientas semánticas que se incorporan en Gazela están destinadas a detectar unos determinados tipos de estructuras lingüísticas que permitan identificar si lo que ha sido publicado puede ser ofensivo. En tal caso, se generarán recomendaciones y/o avisos que serán enviados al usuario. Además, este tipo de análisis se efectúa de forma bidireccional, analizando no sólo lo que un usuario publica en su muro de Facebook, sino también lo que otros usuarios escriben en los muros a los que el usuario tiene acceso. En este caso se informaría a la persona que ha publicado el contenido ofensivo de las posibles consecuencias legales que su acción pudiera acarrearle.

Para la detección de este tipo de irregularidades se están empleando algoritmos basados en diccionarios, que incor- poran reglas semánticas sencillas, de forma que se puedan extraer diferentes tipos de indicios de un texto. Sobre ello se aplican algoritmos inteligentes de clasificación, que son los encargados de determinar si los indicios que han sido detectados en el texto son realmente ofensivos o son propios del lenguaje.

\section{Gazela ayuda a promover y concienciar} sobre las implicaciones éticas y legales de publicar cierta información en las redes sociales u otros medios de difusión

\section{Conclusiones y trabajo futuro}

Gazela es un sistema de concienciación y asesoramiento en el buen uso de las redes sociales a la hora de publicar información y fotografías, especialmente dirigido a las personas más jóvenes. Para ello cuenta con una serie de módulos capaces de identificar a las personas a través de tecnologías biométricas faciales, analizar textos escritos para determinar si en ellos se está publicando información personal de carácter confidencial (teléfonos, direcciones, DNIs, etc.) o si contienen expresiones ofensivas que pudieran ser denunciables ante la ley.

A día de hoy, Gazela está integrada con Facebook mediante la autenticación automática y el acceso a las nuevas publicaciones en texto e imagen. Además comunica a miGuardaespaldas y miAbogado las infracciones, agresiones y datos protegidos encontrados durante el proceso de análisis de la información obtenida.

Con respecto al análisis biométrico, permite la identificación en fotografías de personas previamente registradas. Se sigue trabajando en toda la cadena de procesamiento para superar deficiencias observadas en la detección en diferentes poses y con condiciones de iluminación muy diferenciadas.

Se espera poder realizar una prueba piloto de Gazela en el actual curso académico 2012-2013, para validar su funcionamiento en un entorno real y comprobar qué grado de aceptación tiene por parte de sus futuros usuarios.

Se está trabajando en la mejora de los sistemas de reconocimiento facial, optimizando tanto el algoritmo como los filtros de mejora de imagen, buscando tasas de error bajas en diferentes condiciones ambientales (día, noche, interiores, exteriores, sol, Iluvia...) e integrando Gazela con Tuenti y Twitter.

En el futuro estará accesible como un servicio online de pago al cual se accederá mediante un navegador web.

\section{Agradecimientos}

Este trabajo ha sido elaborado dentro del proyecto Gazela, el asesor digital de los adolescentes, subvencionado por el Departamento de Industria del Gobierno Vasco y el Fondo Europeo de Desarrollo Regional (Feder), dentro del programa Gaitek. 
Queremos agradecer la colaboración de las empresas participantes en el proyecto: Entelgy Ibai, Seinale y el Colegio La Salle de Irún (Gipuzkoa).

Se hace un seguimiento de los contactos de cada usuario, analizando lo que publican en la Red

\section{Recursos y bibliografía}

Chen, Terrence; Yin, Wotao; Zhou, Xiang-Sean; Comaniciu, Dorin; Huang, Thomas. "Illumination normalization for face recognition and uneven background correction using total variation based image models". En: Procs of the IEEE Computer Society conf on computer vision and pattern recognition (CVPR'05), 2005, v. 2, pp. 532-539.

Control parental de Windows7

http://windows.microsoft.com/es-ES/windows7/products/ features/parental-controls

Control parental de Mozilla Firefox

http://www.atareao.es/ubuntu/software-para-tu-ubuntu/ control-parental-con-firefox

ConsumerSearch. Parental Control Software Reviews http://www.consumersearch.com/parental-control-software

Decálogo de consejos de EMICI

http://www.ciberbullying.com/cyberbullying/2010/09/01/ decalogo-para-una-victima-de-ciberbullying

De-Luis-García, Rodrigo; Alberola-López, Carlos; Aghzout, Otman; Ruiz-Alzola, Juan. "Biometric identification systems". Signal process, 2003, Dec., v. 83, n. 12, pp. 2539-2557. http://dx.doi.org/10.1016/j.sigpro.2003.08.001

Face recognition homepage. Resumen de algoritmos para reconocimiento facial. http://www.face-rec.org/algorithms

Graph API. Librerías de comunicación con la información contenida en Facebook.

http://developers.facebook.com/docs/reference/api/

Kong, Seong; Heo, Jingu; Abidi, Besma; Paik, Joonki; Abidi, Mongi. "Recent advances in visual and infrared face recognition - a review". Computer vision image understanding, 2005 , v. 97 , n. 1 , pp. 103-135.

http://dx.doi.org/10.1016/j.cviu.2004.04.001

Guodong, Guo. "Face recognition by support vector machines". En: Procs. 4th IEEE intl conf on automatic face and gesture recognition, 2000, pp. 196-201.

http://dx.doi.org/10.1109/AFGR.2000.840634

Latha, Ponmary P.; Ganesan, L.; Annadurai, S. "Face recognition using neural networks". Signal processing: an international journal (SPIJ), 2009, v. 3, n. 5, pp. 153-160.

http://www.cscjournals.org/csc/manuscript/Journals/SPIJ/ volume3/Issue5/SPIJ-37.pdf

Nefian, Ara V.; Hayes III, Monson. "Hidden Markov models for face recognition". En: Procs intl conf on acoustics, speech and signal processing, 1998, pp. 2721-2724.

http://www.cse.psu.edu/ rcollins/CSE586Spring2010/ papers/HMMnefianFaceRec.pdf

Net nanny. Software de control parental.

http://www.netnanny.com

Protocolo EMICI. Protocolo de actuación escolar ante el cyberbullying elaborado por EMICI (Equipo Multidisciplinar de Investigación sobre Cyberbullying) para el Gobierno Vasco. http://www.protocolo-ciberbullying.com

Reputation manager. Aplicación para el manejo de la reputación online.

http://www.reputation.com

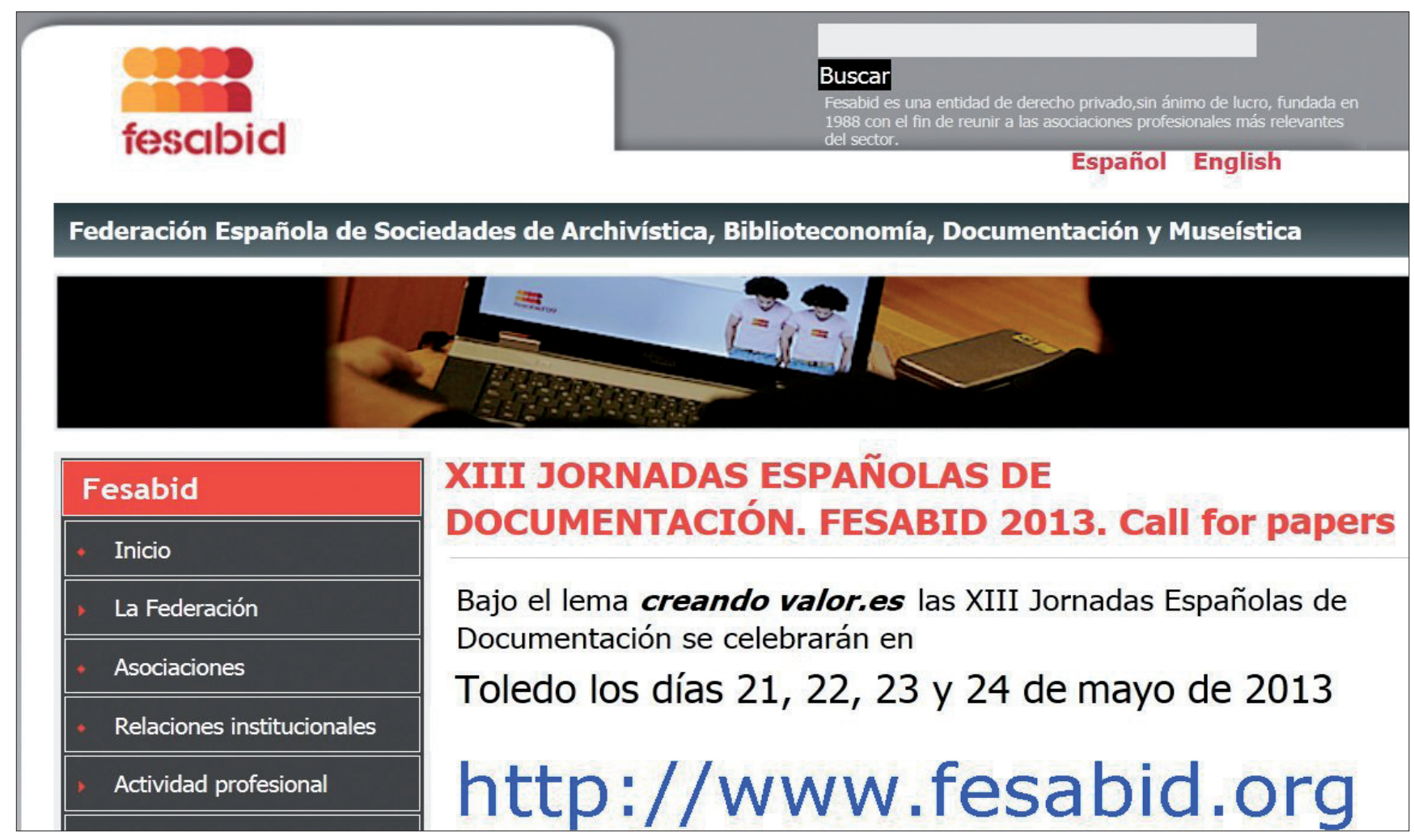

